\title{
Edaravone protects endotoxin-induced liver injury by inhibiting apoptosis and reducing proinflammatory cytokines
}

\author{
L. Zong ${ }^{1,2 *}$, Q.H. Yu ${ }^{3 *}$, Y.X. Du ${ }^{2}$ and X.M. Deng \\ ${ }^{1}$ Department of Anesthesiology, Changhai Hospital, Second Military Medical University, Shanghai, China \\ ${ }^{2}$ Department of Anesthesiology, No. 82 Hospital of People's Liberation Army, Jiangsu, China \\ ${ }^{3}$ Department of Gastroenterology, Changhai Hospital, Second Military Medical University, Shanghai, China
}

\begin{abstract}
Studies have shown that edaravone may prevent liver injury. This study aimed to investigate the effects of edaravone on the liver injury induced by D-galactosamine (GaIN) and lipopolysaccharide (LPS) in female BALB/c mice. Edaravone was injected into mice $30 \mathrm{~min}$ before and $4 \mathrm{~h}$ after GalN/LPS injection. The survival rate was determined within the first $24 \mathrm{~h}$. Animals were killed $8 \mathrm{~h}$ after GalN/LPS injection, and liver injury was biochemically and histologically assessed. Hepatocyte apoptosis was measured by TUNEL staining; proinflammatory cytokines [tumor necrosis factor- $\alpha$ (TNF- $\alpha$ ) and interleukin-6 (IL-6)] in the liver were assayed by ELISA; expression of caspase- 8 and caspase-3 proteins was detected by Western blot assay; and caspase-3 activity was also determined. Results showed that GalN/LPS induced marked elevations in serum aspartate aminotransferase (AST) and alanine aminotransferase (ALT). Edaravone significantly inhibited elevation of serum AST and ALT, accompanied by an improvement in histological findings. Edaravone lowered the levels of TNF- $\alpha$ and IL- 6 and reduced the number of TUNEL-positive cells. In addition, $24 \mathrm{~h}$ after edaravone treatment, caspase-3 activity and mortality were reduced. Edaravone may effectively ameliorate GalN/LPS-induced liver injury in mice by reducing proinflammatory cytokines and inhibiting apoptosis.
\end{abstract}

Key words: Edaravone; Apoptosis; Endotoxin; Liver injury; Inflammation

\section{Introduction}

Acute liver injury results from the massive death of liver cells, leading to the development of hepatic encephalopathy and severe impairment of liver function (1). Acute liver injury still has a high mortality rate, despite significant progress in liver support systems and liver transplantation. A variety of studies have been conducted to investigate the pathogenic mechanism of acute liver injury, and various measures have been taken to treat this disease. Edaravone, a free-radical scavenger, has been found to exert protective effects on injuries to the brain (2), lung (3), and liver (4). Studies have shown that edaravone may effectively ameliorate liver injury induced by D-galactosamine (GaIN)/lipopolysaccharide (LPS) (5) and endotoxin-induced liver injury after partial hepatectomy in rats (6). The protective effects of edaravone are attributed to the direct scavenging of hydroxyl radicals and inhibition of lipoxygenase activity (7).
Inflammation is another major pathogenic mechanism of acute liver injury. In paraquat or carbon tetrachlorideinduced acute liver injury, proinflammatory cytokines [including tumor necrosis factor (TNF)- $\alpha$ and interleukin (IL)-6] increase markedly, but are reduced after antiinflammatory treatment $(8,9)$.

In addition to oxidative stress and inflammation, hepatocellular apoptosis is another important mechanism underlying liver dysfunction and liver failure following LPS treatment $(10,11)$, and it represents an early, general, and possibly causal event in inflammatory liver failure (12). Apoptotic hepatocytes may also act as a signal to trigger neutrophil transmigration and subsequent parenchymal cell attack (13). Thus, apoptotic cell death, even if affecting less than $10 \%$ of hepatocytes, has to be considered as relevant in overall liver injury (14). The present study was undertaken to evaluate the protective

Correspondence: X.M. Deng, Department of Anesthesiology, Changhai Hospital, Second Military Medical University, Shanghai 200433, China. Fax: +86-517-8356-8795. E-mail: dxmxmd1979@163.com

${ }^{*}$ These authors contributed equally to this study.

Received May 8, 2013. Accepted October 22, 2013. First published online January 29, 2014. 
effect of edaravone pretreatment and treatment of liver injury secondary to GalN and LPS exposure, and to determine the influence of edaravone on apoptosis and proinflammatory cytokines in this animal model.

\section{Material and Methods}

\section{Animals and treatment}

A total of 86 female BALB/c mice weighing 18-20 g and 6 weeks of age were purchased from Shanghai SLAC Laboratory Animal Co. Ltd. (China). These animals were housed in cages in a temperature and humidity controlled environment (temperature: $24 \pm 1{ }^{\circ} \mathrm{C}$; humidity: 40-60\%) with a 12:12-h light-dark cycle. All experimental procedures were conducted according to the Guide for the Care and Use of Laboratory Animals developed by the United States National Institutes of Health, and this study was approved by the Ethics Committee for Animal Experiments of the School of Medicine, Shanghai Jiaotong University, China.

Mice were randomly divided into four groups: shamoperated (control), liver injury (LI), $1 \mathrm{mg} / \mathrm{kg}$ edaravone (Eda-1), and $3 \mathrm{mg} / \mathrm{kg}$ edaravone (Eda-3). In the latter three groups, liver injury was induced by intraperitoneal injection of GalN (50 mg; Sigma, USA) and LPS (40 ng, Sigma). In the Eda-1 and Eda-3 groups, mice were intraperitoneally injected with edaravone (Simcere Doyea Pharmaceutical Co., China) 30 min before and $4 \mathrm{~h}$ after GalN/LPS injection. In the LI group, mice were intraperitoneally injected with normal saline of equal volume at the same time points. Mice in the control group had no liver injury and were injected with normal saline of equal volume at corresponding time points.

\section{Detection of liver function and cytokines}

Blood was collected from the orbit at $1.5 \mathrm{~h}$ for TNF- $\alpha$ detection after GalN/LPS treatment and at $8 \mathrm{~h}$ for the detection of IL-6, serum alanine aminotransferase (ALT), and aspartate aminotransferase (AST). IL- 6 and TNF- $\alpha$ were measured with commercially available kits (R\&D Systems, USA) according the manufacturer's instructions. Serum ALT and AST were determined with a HITACHI 7020 autoanalyzer, using commercially available reagents (Shino-test Corporation, Japan).

\section{Determination of survival rate, histological examination, and TUNEL staining}

The survival rate was monitored within the first $24 \mathrm{~h}$ after GalN/LPS injection. For histological examination, animals were anesthetized at $8 \mathrm{~h}$ after GaIN/LPS treatment and $4 \%$ neutral formalin in phosphate-buffered saline was perfused via the hepatic portal vein. The liver was removed and embedded in paraffin followed by hematoxylin and eosin staining. The criteria for scoring hepatocellular necrosis were: 0 , normal tissue; 1 , cells with degeneration, edema, and cytoplasmic loose mesh;
2, scattered spotty necrosis; 3 , scattered areas of massive necrosis; 4 , massive necrosis over a large area $(\geqslant 1 / 4$ hepatic lobule); and 5 , extensive necrosis $(\geqslant 1 / 2$ hepatic lobule). Five fields were randomly selected from each section at a magnification of $200 \times$ for histological evaluation. Pathological examination was done by two independent pathologists blinded to this study, and consensus was obtained. In addition, TUNEL staining (R\&D Systems) was done to detect apoptotic cells according to the manufacturer's instructions. TUNELpositive cells were counted in 10 randomly selected fields at a high magnification. Data are reported as the number of TUNEL-positive cells per field.

\section{Western blot assay}

Animals were killed by an overdose of anesthesia $8 \mathrm{~h}$ after GalN/LPS injection and the livers were collected. Tissues were lysed in a lysis buffer (10X, \#9803; Cell Signaling Technology, USA) according to the manufacturer's instructions, followed by extraction of total protein. After determination of protein concentration, equal amounts of protein were loaded, separated by $10 \%$ sodium dodecyl sulfate-polyacrylamide gel electrophoresis, and transferred electrophoretically to nitrocellulose membranes. Membranes were blocked with $2 \%$ bovine serum albumin (BSA) in Tris-buffered saline containing $0.1 \%$ Tween 20 (TBST) at room temperature for $1 \mathrm{~h}$ and then incubated overnight at $4^{\circ} \mathrm{C}$ with TNF receptor 1 (TNF-R1), TNF receptor 2 (TNF-R2), caspase-3 or cleaved caspase-3 antibody (1:1000; Cell Signaling Technology), and $\beta$-actin (1:1000; Sigma) in $2 \%$ BSA in TBST. Horseradish peroxidase-conjugated anti-mouse or anti-rabbit secondary antibody (Santa Cruz Biotechnology, USA) in $2 \%$ BSA in TBST $(1: 2000)$ was used to treat these membranes for $1 \mathrm{~h}$, followed by visualization with an enhanced chemiluminescence detection kit (Amersham; GE Healthcare Life Sciences UK). Bands were scanned using a densitometer (GS-700; Bio-Rad Laboratories, USA), and quantification was performed using the Multi-Analyst 1.0.2 software (Bio-Rad).

\section{Detection of caspase-3 activity}

Liver tissues were homogenized in a buffer, $\mathrm{pH} 7.5$, containing $25 \mathrm{mM}$ HEPES, $5 \mathrm{mM} \mathrm{MgCl}$, $5 \mathrm{mM}$ EDTA, $2 \mathrm{mM}$ dithiothreitol, $0.1 \%$ CHAPS, $0.5 \mathrm{mM}$ Pefabloc (Roche Molecular Biochemicals, USA), $0.1 \mathrm{mg} / \mathrm{mL}$ leupeptin, and $0.1 \mathrm{mg} / \mathrm{mL}$ pepstatin. The homogenates were centrifuged at $12,000 \mathrm{~g}$ for $15 \mathrm{~min}$ at $4^{\circ} \mathrm{C}$, followed by collection of the supernatant. Then, $50 \mathrm{~g}$ protein in the supernatant was assayed for caspase- 3 activity, which was measured with the ApoAlert caspase-3 colorimetric assay kit (BD Biosciences, USA) according to the manufacturer's instructions.

\section{Statistical analysis}

Statistical analysis was performed with the SPSS 
version 13.0 statistics software (SPSS Inc., USA). Quantitative data are reported as means \pm SD. Statistical analysis was done with one-way analysis of variance (ANOVA) followed by the Student-NewmanKeuls test. A value of $\mathrm{P}<0.05$ was considered to be statistically significant. Survival time was analyzed with a survival curve analysis.

\section{Results}

\section{ALT, AST, and survival rate}

Serum ALT and AST levels in the control group were $36.6 \pm 5.5$ and $29.5 \pm 4.9 \mathrm{U} / \mathrm{L}$, respectively. The serum ALT and AST levels increased by approximately 156- and 40-fold, respectively, at $8 \mathrm{~h}$ after GalN/LPS injection compared with controls (Figure $1 \mathrm{~A}$ and $\mathrm{B}$ ). This suggests that GalN/LPS treatment successfully induced acute hepatic injury. Mice without edaravone treatment began to die within $6 \mathrm{~h}$ after GaIN/LPS injection, and the mortality rate was $80 \%$ at $24 \mathrm{~h}$. However, pretreatment with edaravone markedly reduced mortality. The survival rate was about $40 \%$ in the Eda-1 group and about $80 \%$ in the Eda-3 group, which were both significantly higher than that in the control group $(\mathrm{P}<0.05$; Figure $1 \mathrm{C})$.

\section{Histopathology and apoptosis}

The above findings were supported by those obtained by morphological examination of the liver. At $8 \mathrm{~h}$ after GalN/ LPS injection (LI group), multifocal, midzonal hepatocellular necrosis and severe hemorrhage were observed. In the liver of animals with edaravone treatment, the size and frequency of necrotic foci were significantly reduced compared with the LI group (Figure 2A). The hepatocellular necrosis score was significantly decreased from $4.3 \pm 0.4$ in the LI group to $2.9 \pm 0.4$ in the Eda-3 group. However, there was no significant difference between the scores in the Eda-1 $(3.8 \pm 0.5)$ and $\mathrm{LI}$ groups $(4.3 \pm 0.4)$, although a slight reduction was observed in the Eda-1 group $(P>0.05)$. Apoptotic hepatocytes were detected by TUNEL staining. A large number of TUNEL-positive hepatocytes (183 \pm 24 cells/field) were observed in the liver at $8 \mathrm{~h}$ after GalN/ LPS treatment. However, the number of TUNEL-positive hepatocytes was markedly reduced in the livers of edaravone-treated animals $(153 \pm 30$ and $97 \pm 21$ cells/field, respectively; Figure $2 \mathrm{~B}$ and $\mathrm{C}$ ).

\section{Proinflammatory cytokines}

Our preliminary study showed that TNF- $\alpha$ levels increased significantly as early as $1.5 \mathrm{~h}$ after GalN/LPS treatment. Thus, TNF- $\alpha$ levels were measured at $1.5 \mathrm{~h}$ after GalN/LPS injection, although edaravone treatment was not performed. Edaravone at $3 \mathrm{mg} / \mathrm{kg}$ significantly decreased TNF- $\alpha$ levels from $2759.1 \pm 974.0 \mathrm{ng} / \mathrm{mL}$ in the LI group to $1797.0 \pm 396.9 \mathrm{ng} / \mathrm{mL}$, while TNF- $\alpha$ remained unchanged in the Eda-1 group after edaravone treatment, and was comparable between $\mathrm{LI}$ and Eda-1 groups (Figure $3 \mathrm{~A}$ ). In addition, edaravone at 1 or $3 \mathrm{mg} / \mathrm{kg}$ significantly reduced serum IL-6 levels at $8 \mathrm{~h}$ after GalN/ LPS injection (Figure 3B).

\section{Expression of caspase- 8 and caspase- 3 proteins and caspase-3 activity}

Western blot assay demonstrated that expression of full-length caspase-8 and caspase- 3 was reduced in mice undergoing GalN/LPS treatment, but expression of cleaved caspase- 8 and caspase- 3 increased significantly in the LI and Eda groups. However, this elevation was significantly lower in the Eda groups (especially the Eda-3 group) compared with the LI group (Figure 4A and B). Caspase-3 activity in the LI group was increased 2.8-fold compared with controls, but this increase was lower in both the Eda-1 and Eda-3 groups (Figure 4B; 2.2- and 1.5-fold, respectively, Figure 5). TNF-R expression remained unchanged among the three groups (Figure 4C).

\section{Discussion}

Traditionally, hepatocyte necrosis is a characteristic feature of fulminant hepatic failure, but increasing evidence indicates a dominant role of hepatocyte apoptosis in the
A

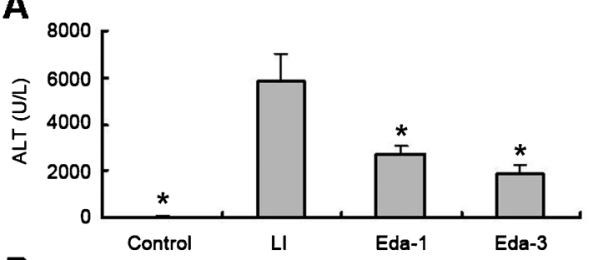

B

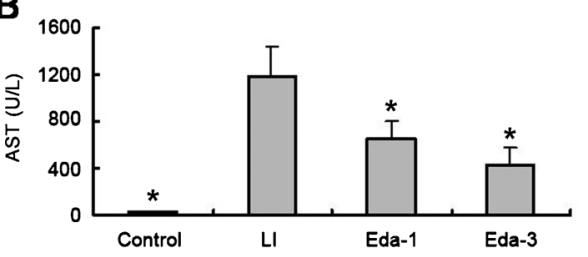

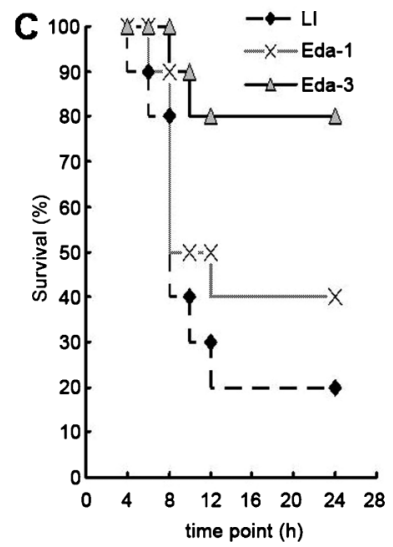

Figure 1. Liver enzymes and survival rate in the different groups. A, Serum alanine aminotransferase (ALT) level $(n=8)$. B, Serum aspartate aminotransferase (AST) level $(n=8)$. $C$, Survival rate of mice at different time points after GalN/ LPS treatment $(n=10)$. LI group: liver injury induced by D-galactosamine and lipopolysaccharide (GalN/LPS); Eda-1 and Eda-3 groups: edaravone at 1 and $3 \mathrm{mg} / \mathrm{kg} 30 \mathrm{~min}$ before and $4 \mathrm{~h}$ after GalN/LPS-induced liver injury. Data are reported as means $\pm \mathrm{SD}$. ${ }^{*} \mathrm{P}<0.05$ vs $\mathrm{LI}$ group (ANOVA). 
A

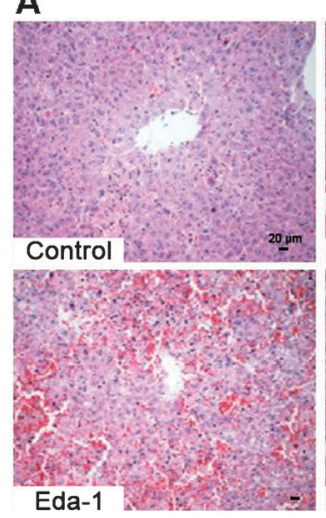

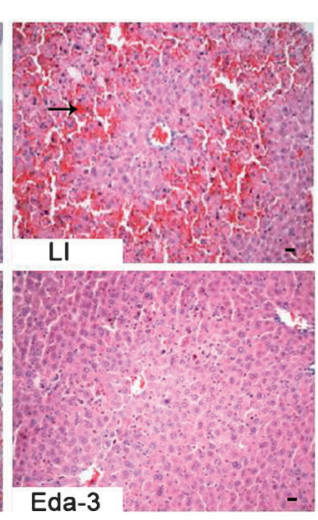

B

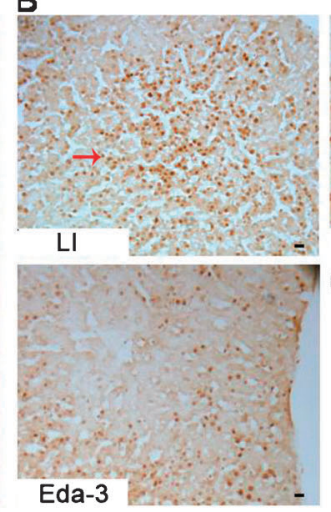

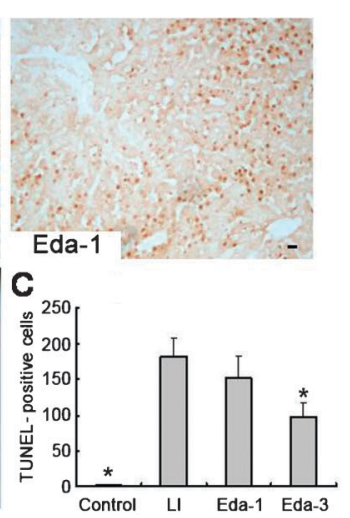

Figure 2. Histological examination and TUNEL staining. A, H\&E staining of the livers after D-galactosamine and lipopolysaccharide (GalN/LPS) treatment. Representative images were captured from the 4 groups (scale bar: $20 \mu \mathrm{m}$ ). Sham group: normal lobular architecture and cell structure; LI group: GalN/LPS induced extensive hemorrhage (black arrow), massive hepatocellular necrosis and a moderate increase in inflammatory cells; Eda-1 and Eda-3 groups: edaravone at 1 and $3 \mathrm{mg} / \mathrm{kg}$ ameliorated GalN/LPS-induced liver injury. $B$, TUNEL staining of hepatocytes (red arrow; scale bar: $20 \mu \mathrm{m}$ ). $C$, Number of apoptotic hepatocytes. Data are reported as means $\pm S D(n=6) .{ }^{*} \mathrm{P}<0.05$ vs LI group (ANOVA).

pathogenesis of fulminant hepatic failure (11). In the present study, liver injury was induced in rats by administration of GalN/LPS, and was then followed by pretreatment and treatment with edaravone. Our results showed that edaravone treatment resulted in reductions in caspase- 3 activity, expression of cleaved caspase- 8 and caspase- 3 proteins, the number of apoptotic cells, and caspase- 3 activity. These effects were accompanied by
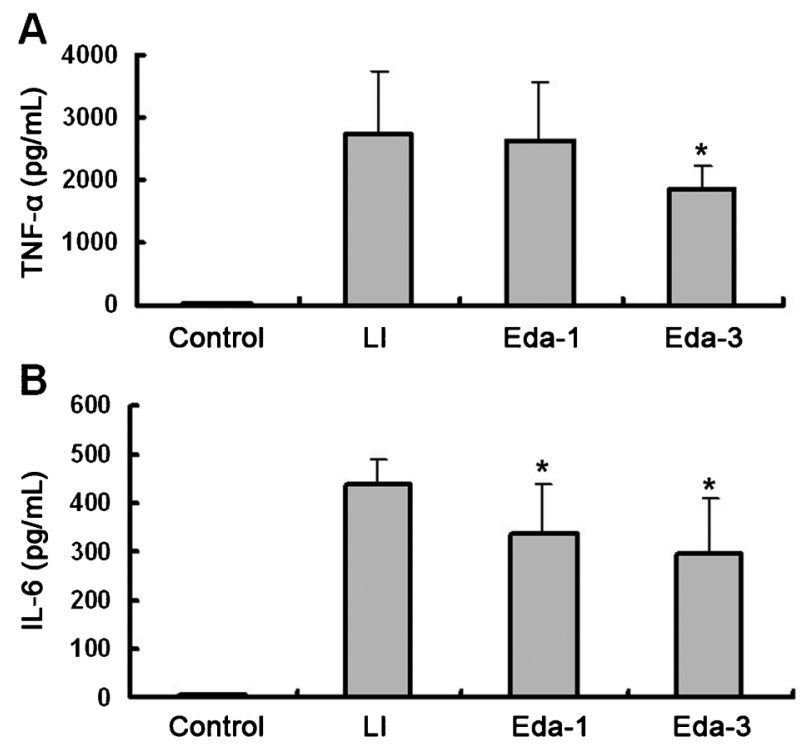

Figure 3. Serum TNF- $\alpha$ and IL-6 levels. LI group: liver injury induced by D-galactosamine and lipopolysaccharide (GalN/LPS); Eda-1 and Eda-3 groups: edaravone at 1 and $3 \mathrm{mg} / \mathrm{kg} 30 \mathrm{~min}$ before and $4 \mathrm{~h}$ after GalN/LPS-induced liver injury. Data are reported as means $\pm S D(n=6)$. ${ }^{*} P<0.05$ vs LI group (ANOVA). decreases in serum TNF- $\alpha$ and IL-6. As a consequence, liver injury was reduced, as indicated by decreased ALT and AST levels, and mortality was reduced within the first $24 \mathrm{~h}$ following GaIN/LPS challenge.

The mechanism of LPS-mediated apoptosis is not well understood. It has been found that LPS-induced apoptosis and proinflammatory cytokines (such as TNF- $\alpha$ ) mediate acute liver failure and septic shock. TNF- $\alpha$ is an important mediator of LPS-induced hepatotoxicity and is involved in LPS-induced liver injury (15). TNF- $\alpha$ induces cell apoptosis via the death domain motif of its receptor, TNF-R (16). Binding of TNF- $\alpha$ to TNF-R may activate caspase-8 to trigger the apoptosis cascade. In our preliminary study, GalN/LPS increased serum TNF- $\alpha$ levels, which reached a maximal level at around $1.5 \mathrm{~h}$ after GalN/LPS treatment and returned to normal within $4 \mathrm{~h}$ (data not shown). In our study, although TNF- $\alpha$ increased markedly, expression of TNF-R remained unchanged. Thus, we speculated that the massive hepatocyte apoptosis in GaIN/LPS-challenged livers was mediated by binding of increased TNF- $\alpha$ to its receptors on the cell surface. In addition, edaravone at $3 \mathrm{mg} / \mathrm{kg}$ significantly decreased TNF- $\alpha$ but had no effect on expression of TNF-R1 and TNF-R2. This suggests that the effect of edaravone was not mediated by a direct decrease of TNF- $\alpha$. In addition, edaravone, even at a high dose, failed to completely reduce the expression of cleaved caspase-8, suggesting that other pathways in addition to the caspase-dependent pathway are involved in TNF- $\alpha$-induced apoptosis.

It has been reported that a variety of extracellular and intracellular death stimuli, such as reactive oxygen species (ROS), trigger apoptosis in a mitochondriadependent pathway (17), and LPS has been confirmed 


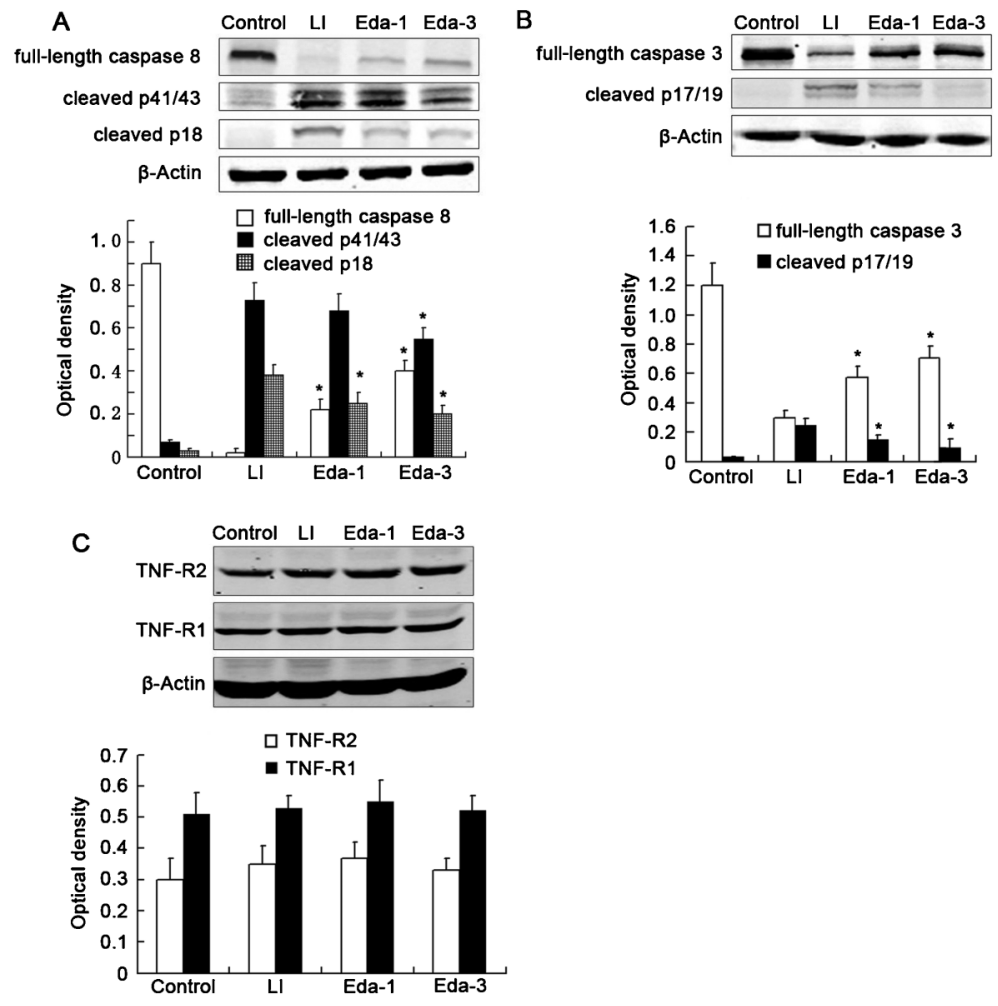

Figure 4. Western blot assay of caspase-3, caspase-8 and TNF-R. LI group: liver injury induced by $\mathrm{D}$-galactosamine and lipopolysaccharide (GalN/LPS); Eda-1 and Eda-3 groups: edaravone at 1 and $3 \mathrm{mg} / \mathrm{kg} 30 \mathrm{~min}$ before and $4 \mathrm{~h}$ after GalN/LPS-induced liver injury. Data are representative of three independent experiments $(\mathrm{n}=8) .{ }^{*} \mathrm{P}<0.05$ vs LI group (ANOVA).

to induce ROS generation (18). The ROS-scavenging property of edaravone has been well documented in numerous previous studies $(19,20)$. LPS-induced ROS activation of an intrinsic mitochondrion-dependent pathway has been confirmed in human alveolar epithelial A549 cells (21), and studies have also shown that edaravone can

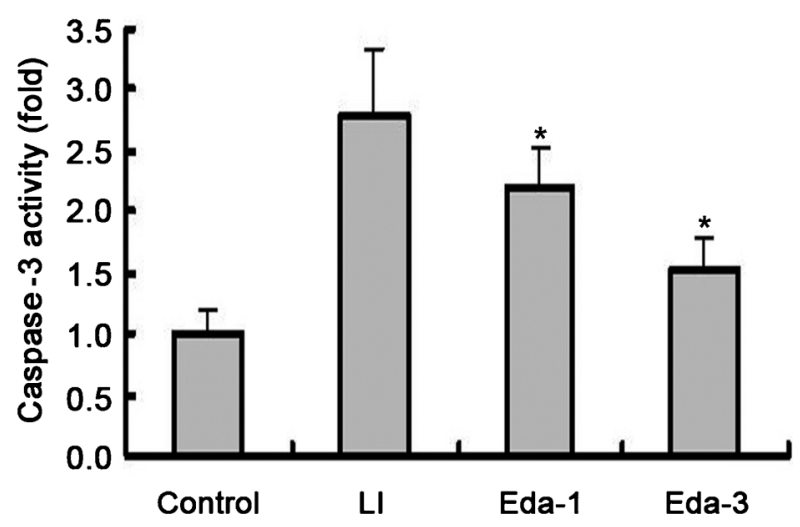

Figure 5. Assay of caspase 3 activity. LI group: liver injury induced by D-galactosamine and lipopolysaccharide (GalN/LPS); Eda-1 and Eda-3 groups: edaravone at 1 and $3 \mathrm{mg} / \mathrm{kg} 30 \mathrm{~min}$ before and $4 \mathrm{~h}$ after GalN/LPS-induced liver injury. Data are representative of three independent experiments $(n=8)$. ${ }^{*} \mathrm{P}<0.05$ vs LI group (ANOVA) inhibit apoptosis caused by liver ischemia/reperfusion injury (22). Thus, it would be of interest to test this hypothesis in a future study by investigating mitochondriadependent pathways (e.g., cytochrome c or caspase-9) in the protective effect of edaravone on liver injury.

Ito et al. (5) also investigated the protective effects of edaravone on acute liver injury. However, the time points of treatment in this study were different from those in the study of Ito et al. Moreover, they investigated the influence of edaravone as a free radical scavenger during oxidative stress. In this study, we emphasized the role of apoptosis in the hepatoprotective effects of edaravone. Our results demonstrated that edaravone can effectively ameliorate GalN/LPS-induced liver injury in mice by reducing proinflammatory cytokines and inhibiting apoptosis.

\section{References}

1. Day HL, Taylor RM. The liver. Part 5: acute liver failure. Nurs Times 2006; 102: 26-27.

2. Kawasaki T, Ishihara K, Ago Y, Nakamura S, Itoh S, Baba $A$, et al. Protective effect of the radical scavenger edaravone against methamphetamine-induced dopaminergic neurotoxicity in mouse striatum. Eur J Pharmacol 2006; 542: 92-99, doi: 10.1016/j.ejphar.2006.05.012.

3. Yang T, Mao YF, Liu SQ, Hou J, Cai ZY, Hu JY, et al. Protective effects of the free radical scavenger edaravone on acute pancreatitis-associated lung injury. Eur $J$ 
Pharmacol 2010; 630: 152-157, doi: 10.1016/j.ejphar. 2009.12.025.

4. Okatani Y, Wakatsuki A, Enzan H, Miyahara Y. Edaravone protects against ischemia/reperfusion-induced oxidative damage to mitochondria in rat liver. Eur J Pharmacol 2003; 465: 163-170, doi: 10.1016/S0014-2999(03)01463-8.

5. Ito K, Ozasa H, Noda Y, Arii S, Horikawa S. Effects of free radical scavenger on acute liver injury induced by $d$ galactosamine and lipopolysaccharide in rats. Hepatol Res 2008; 38: 194-201.

6. Tsuji K, Kwon AH, Yoshida H, Qu Z, Kaibori M, Okumura T, et al. Free radical scavenger (edaravone) prevents endotoxininduced liver injury after partial hepatectomy in rats. $\mathrm{J} \mathrm{Hepatol}$ 2005; 42: 94-101, doi: 10.1016/j.jhep.2004.09.018.

7. Watanabe K, Ma M, Wen J, Kodama M, Aizawa Y. Effects of edaravone in heart of aged rats after cerebral ischemiareperfusion injury. Biol Pharm Bull 2007; 30: 460-464, doi: 10.1248/bpb.30.460.

8. Amirshahrokhi K, Bohlooli S. Effect of methylsulfonylmethane on paraquat-induced acute lung and liver injury in mice. Inflammation 2013; 36: 1111-1121, doi: 10.1007/ s10753-013-9645-8.

9. Shin DS, Kim KW, Chung HY, Yoon S, Moon JO. Effect of sinapic acid against carbon tetrachloride-induced acute hepatic injury in rats. Arch Pharm Res 2013; 36: 626-633, doi: 10.1007/s12272-013-0050-5.

10. Kuhla A, Eipel C, Siebert N, Abshagen K, Menger MD, Vollmar B. Hepatocellular apoptosis is mediated by TNFalpha-dependent Fas/FasLigand cytotoxicity in a murine model of acute liver failure. Apoptosis 2008; 13: 1427-1438, doi: 10.1007/s10495-008-0269-7.

11. Jaeschke H, Gujral JS, Bajt ML. Apoptosis and necrosis in liver disease. Liver Int 2004; 24: 85-89, doi: 10.1111/j.14783231.2004.0906.x.

12. Leist M, Gantner F, Bohlinger I, Tiegs G, Germann PG, Wendel A. Tumor necrosis factor-induced hepatocyte apoptosis precedes liver failure in experimental murine shock models. Am J Pathol 1995; 146: 1220-1234.

13. Lawson JA, Fisher MA, Simmons CA, Farhood A, Jaeschke $\mathrm{H}$. Parenchymal cell apoptosis as a signal for sinusoidal sequestration and transendothelial migration of neutrophils in murine models of endotoxin and Fas-antibody-induced liver injury. Hepatology 1998; 28: 761-767, doi: 10.1002/ hep. 510280324 .

14. Schafer T, Scheuer C, Roemer K, Menger MD, Vollmar B. Inhibition of p53 protects liver tissue against endotoxininduced apoptotic and necrotic cell death. FASEB J 2003; 17: 660-667, doi: 10.1096/fj.02-0774com

15. Bohlinger I, Leist M, Gantner F, Angermuller S, Tiegs G, Wendel A. DNA fragmentation in mouse organs during endotoxic shock. Am J Pathol 1996; 149: 1381-1393.

16. Tartaglia LA, Rothe M, Hu YF, Goeddel DV. Tumor necrosis factor's cytotoxic activity is signaled by the p55 TNF receptor. Cell 1993; 73: 213-216, doi: 10.1016/00928674(93)90222-C.

17. Thornberry NA. Caspases: key mediators of apoptosis. Chem Biol 1998; 5: R97-R103, doi: 10.1016/S10745521(98)90615-9.

18. Maitra U, Singh N, Gan L, Ringwood L, Li L. IRAK-1 contributes to lipopolysaccharide-induced reactive oxygen species generation in macrophages by inducing NOX-1 transcription and Rac1 activation and suppressing the expression of antioxidative enzymes. J Biol Chem 2009; 284: 35403-35411, doi: 10.1074/jbc.M109.059501.

19. Banno M, Mizuno T, Kato H, Zhang G, Kawanokuchi J, Wang $\mathrm{J}$, et al. The radical scavenger edaravone prevents oxidative neurotoxicity induced by peroxynitrite and activated microglia. Neuropharmacology 2005; 48: 283-290, doi: 10.1016/j.neuropharm.2004.10.002.

20. Abe T, Unno M, Takeuchi H, Kakita T, Katayose Y, Rikiyama $\mathrm{T}$, et al. A new free radical scavenger, edaravone, ameliorates oxidative liver damage due to ischemia-reperfusion in vitro and in vivo. J Gastrointest Surg 2004; 8: 604-615, doi: 10.1016/ j.gassur.2004.02.011.

21. Chuang CY, Chen TL, Cherng YG, Tai YT, Chen TG, Chen RM. Lipopolysaccharide induces apoptotic insults to human alveolar epithelial A549 cells through reactive oxygen species-mediated activation of an intrinsic mitochondriondependent pathway. Arch Toxicol 2011; 85: 209-218, doi: 10.1007/s00204-010-0585-x.

22. Shimoda M, Iwasaki Y, Okada T, Kubota K. Edaravone inhibits apoptosis caused by ischemia/reperfusion injury in a porcine hepatectomy model. World J Gastroenterol 2012; 18: 3520-3526, doi: 10.3748/wjg.v18.i27.3520. 